\title{
Virtual Institute for the Modelling of Industrial Manufacturing Systems:
}

Integration of on-line and 'face-to-face' learning in an international platform for teaching and research

Sergio Cavalieri, Marco Garetti, Marco Macchi, Alessandro Campi, Francesca Collina, Alberto Colorni, Ignazio Locatelli, Tiziana Longeri, Gregor Von Cieminski, Jens Lopitzsch, Maria di Mascolo, Yannick Frein, Botond Kadar, Laszlo Monostori, Elisabeta Ilie Zudor, Alberto Meroni and Nicoletta Santambrogio

DIG, Politecnico di Milano, Italy; METID, Politecnico di Milano, Italy; IFA, University of Hanover, Germany; LAG-INPG, Grenoble, France; SZTAKI, Budapest, Hungary; ENFAPI Briantea

Abstract: The aim of an ongoing research project carried out by six European research and educational institutions is to establish a Virtual Institute for the Modelling of Industrial Manufacturing Systems (VIMIMS). The VIMIMS virtual institute has been designed to become an international platform for teaching and research in the field of analysis, design and performance evaluation of industrial manufacturing systems. VIMIMS is implemented as an internetbased portal that offers students and researchers from around the globe opportunities to study, teach, research and communicate. This paper aims to describe the architecture of the VIMIMS web portal and its utilisation by users from a real educational institute. This platform will be integrated into courses of project partners and will act as a supplement to ordinary 'face-to-face' lectures and practical activities.

Key words: virtual institute, modelling, industrial manufacturing systems

\section{INTRODUCTION}

The objective of the VIMIMS project is "to establish a virtual institute specifically focused on knowledge sharing and integration of competencies in the educational and research area of modelling and design of industrial

The original version of this chapter was revised: The copyright line was incorrect. This has been corrected. The Erratum to this chapter is available at DOI: 10.1007/978-0-387-35615-0_52 
manufacturing systems" (proposal submitted to Minerva action of the European Commission, March 2000).

The design solution of the VIMIMS environment is not to completely replace 'face-to-face' learning, but to support it with distance learning components, thus trying to integrate benefits of both traditional and on-line learning modes. In the VIMIMS consortium intention, this 'pragmatic' formula is proposed for 'managing complexity' arising from different learning contexts. To this end, a high degree of flexibility is left to VIMIMS users for selecting the most appropriate educational approach in their educational context. Moreover, this 'pragmatic' approach serves to deliver on-line teaching courses as part of official teaching courses, i.e. to deal with the constraints and rules of the real-life operation of each educational institute that will adopt the VIMIMS web portal.

\section{THE VIMIMS DESIGN STRATEGY}

The VIMIMS virtual institute is designed to become an international platform for teaching and research in the field of analysis, design and performance evaluation of industrial manufacturing systems and it is implemented as an internet-based portal that offers students and researchers from around the globe the opportunity to study, teach, research and communicate on this subject. Therefore the VIMIMS web portal will include educational material and a knowledge base on the subject of the 'Modelling of Industrial Manufacturing Systems (MIMS)' supplementing standard 'faceto-face' teaching in real educational institutes.

The VIMIMS consortium will test this integrated learning scheme in the context of different educational institutions, ranging from universities (i.e. IFA, LAG-INPG and Politecnico di Milano) and research centres (i.e. SZTAKI) to secondary schools (i.e. ENFAPI Briantea). The VIMIMS partners will use the VIMIMS web portal to supplement official teaching courses offered within their own organisation and to their own students (with different profiles in terms of educational background and knowledge base).

While developed and tested in a university environment, the project also addresses industry needs. In fact in the last few years an increasing interest in distance learning technology has been seen in the industrial world: this is easily explained by the need for a continuing education in order to maintain the skills of employees and to help the merging of knowledge acquired in different places. Other potential users of this environment may be everyone interested in independently increasing their skills.

An important aspect of innovation of the VIMIMS approach is the objective to adapt the technology to the user. In fact in many cases learning platforms are 'fixed' and force the user to adapt to them. Using the 
experience of the METID partner on teaching technologies, VIMIMS is designed and is flexible enough to support different 'educational styles', following users' requests or particular teacher's needs. Another important characteristic of VIMIMS is the possibility for teachers to build their own courses using different pre-existent learning objects.

To achieve these results a special design solution was selected: i.e. not to provide, inside VIMIMS, full (monolithic) 'blocks of education' (i.e. lessons, tutorials and so on), but to establish a repository of small bricks of knowledge (i.e. learning objects, so called ELM, Elementary Learning Modules in VIMIMS) and leave to the teacher (through an instant web editor called 'Easy-WEB') the possibility to pre-assemble his/her own course, specifically tailored to his/her classroom and to his/her preference.

\section{LEARNING IN VIMIMS}

The characteristics defining the VIMIMS design strategy are embedded in services. Traditional web sites of schools and universities only offer content. Instead, VIMIMS tries to unify services to deliver content and services, enforcing the collaboration amongst users (students and teachers). Both synchronous (for example, chat) or asynchronous (for example, forum) collaboration will be used for experimentation in VIMIMS: collaborative learning (synchronous) refers to situations 'in which two or more subjects build synchronously and interactively a joint solution to some problem', while co-operative learning (asynchronous) corresponds to 'a protocol in which the task is in advance split into sub-tasks that the partners solve independently' (Dillenbourg and Schneider, 1998). In the VIMIMS project, collaborative learning will mostly be tested in theoretical learning and practical laboratory activities. Co-operative learning will be tested between trans-national groups of students carrying out projects in co-operation with industrial companies and using the software tools made available by the VIMIMS virtual laboratory.

\subsection{Integrated on-line and face-to-face learning}

In Curtis and Lawson (2001) evidence of good quality interactions amongst students not present in a unique physical site, but interacting from remote locations, emerges from experimentation and analysis on students' on-line exchanges. However, a shared opinion as to whether interaction in on-line learning environments brings benefits to learning activities has not been reached yet. In fact, adoption of on-line tools might bring some disadvantages: interactions amongst students are mediated through on-line 
tools; furthermore there is an absence of non-verbal cues and text-on-screen is a very limited mode to express semantically-rich exchanges.

As stated, the VIMIMS project will combine integrated 'face-to-face' learning and on-line learning. Basically, on-line tools will be adopted:

- to enhance collaborative learning and the efficiency of 'student-toteacher' and 'teacher-to-teacher' interaction during theoretical learning and practical laboratory activities;

- to enhance co-operation in groups of students working on the same project from different educational institutes, i.e. to provide them with the means to communicate, organise and execute project activities from remote locations.

The experimentation will allow the testing of this 'pragmatic' learning environment and to evaluate whether the extent of the adoption of on-line tools depends on the learning activity and what is the 'best' extent of adoption of on-line tools during theoretical learning, practical laboratory activities and remote project working.

\subsection{Actors in the learning process}

Teachers and students are the main actors who will use the VIMIMS web portal in educational activities in each VIMIMS partner organisation.

From a functional point of view, i.e. from the point of view of functions offered by the web portal implementing it, the following different kind of users are to be considered:

- teachers: VIMIMS teachers are responsible for creating on-line courses in the VIMIMS web portal, for administering the courses they are offering and for holding teaching courses using the VIMIMS educational institute.

- content providers: teachers (and teachers' tutors) are also responsible for the provision of educational material (modules) for teaching courses and for finding references to be put in the VIMIMS virtual institute library; furthermore they decide which software tools should be made available in the VIMIMS virtual laboratory and are responsible for configuring and administering these tools during their usage; thus they act as content providers in this role.

- students and laboratory users: students are the VIMIMS learners; they attend theoretical lectures on MIMS techniques and carry out practical laboratory activities in the virtual institute; they might also gather and integrate knowledge by means of any additional educational material and reference available in the virtual institute library.

- visitors: they are students, teachers, and researchers visiting the VIMIMS web-site. 


\section{VIMIMS ARCHITECTURE}

\subsection{VIMIMS sections}

The concept of the VIMIMS virtual institute calls for a coherent analogy with a real institute. This is the main design principle in the creation of the architecture for the VIMIMS web portal site. The VIMIMS web portal site provides two main 'views' of the VIMIMS virtual institute.

The 'user view' is structured in four sections: the lecture theatres is the virtual environment where teachers hold VIMIMS on-line courses and students attend them; the laboratory is the virtual environment where students (or other laboratory users) use VIMIMS software tools; the library is the virtual environment where references and scientific content on the subject of MIMS are collected; the virtual factory is the environment where virtual tours of industrial factories can take place, i.e. educational institutes might organise visits of real industrial factories, that are collected in this virtual environment. The 'Content provider view' is a service view for building and managing the VIMIMS learning contents: it is the virtual environment where content providers design and deliver contents on MIMS subjects. The content provider view is structured in one section, i.e. the Content provider's workshop.

\subsection{VIMIMS modes of access}

The VIMIMS virtual institute is accessed by means of the VIMIMS web portal homepage (http://www.vimims.org). The homepage provides services to access as a registered or as an anonymous user: students, teachers, content providers and laboratory users have a personalised log-in to access as registered users; visitors enter as guests and they have only limited access to VIMIMS sections. Each user profile has his/her own access rights and 'views' of the VIMIMS virtual institute. VIMIMS web pages are dynamically generated to provide educational content and services in a usersensitive way.

\subsection{VIMIMS services}

The VIMIMS portal provides two types of services. Communication and interaction services are provided mostly to support students in their learning activity: communication services are the show case and the agenda; interaction services are the chat, forum and messenger. Other support services are provided to help content providers and teachers in designing, organising, delivering and administering educational material: they include 
administration services for supporting a teacher in the on-line administration of the course he/she is holding and content provision services for supporting teachers and content providers in the design and delivery of course content.

\section{CONCLUSIONS AND FUTURE DEVELOPMENTS}

An outline of the VIMIMS project has been presented. The project is currently in its second year of development:

- the architecture of the virtual institute has been fully defined and the corresponding educational processes and functions have been analysed;

- the design and the implementation of the software platform (web portal) is fully finished and the beta-version of the platform is already running;

- the content of VIMIMS (is made of formal descriptive methods such as those described by Bartolotta, Corradi and Garetti (1999) and Menzel and Mayer (1998), analytical methods such as those of Dallery, David and Xie (1989), and Di Mascolo, David and Dallery (1991). Content from Nyhuis (1994), Wiendahl (1987) and the simulation methods of Carrie (1988) are under development;

- the first courses, based on the VIMIMS platform, are due for use in spring 2002: official courses will be delivered regularly by the end of the project (i.e. the end of 2002).

Main issues of the project are as follows:

- the organisation of the VIMIMS virtual institute follows a coherent analogy with a real institute: sections of the platform are lecture theatres, laboratory, library and virtual factory; users of the virtual institute are content providers, teachers, students, laboratory users and visitors;

- the design of the VIMIMS software platform is based on a flexible organisation of content structure (modularisation) and on flexible content management, thus allowing 'personalised' course building by teachers;

- a combination of 'on-line' and 'face-to-face' educational modes is provided in order to achieve the most convenient mixture;

- an international dimension of collaborative and co-operative learning schemes is provided, thus allowing students from different countries to study the same course or to remotely co-operate on the same project.

After practising the VIMIMS platform in official courses of partner institutions, the VIMIMS consortium intends to exploit the potential of the platform in two different directions: i) towards industry needs for continuing education and for personnel training, ii) to the subjects of operations and logistics management as an enlargement of platform focus. 


\section{REFERENCES}

Bartolotta, A., Corradi, E. and Garetti, M. (1999) Developing an ontology for the modelling of manufacturing systems. In Proceedings of IFIP International Enterprise Modelling Conference IEMC 99, Verdal, Norway.

Curtis, D.D. and. Lawson, M.J. (2001) Exploring collaborative on-line learning. JALN, 5, 1

Dallery, Y., David, R. and Xie, X.L. (1989) Approximate analysis of transfer lines with unreliable machines and finite buffers. IEEE Transactions on Automatic Control, 34, 943953

Di Mascolo, M., David, R. and Dallery, Y. (1991) Modelling and analysis of assembly systems with unreliable machines and finite buffers. IIE Transactions, 23, (4), 315-330

Dillenbourg, P. and Schneider, D. (1998) Collaborative learning and the internet. (Available at: http://tecfa.unige.ch/tecfa/research/CMC/colla/iccai95_1.html, August, 1998.)

Menzel, C. and Mayer, R.J. (1998) The IDEF family of languages. In P. Bernus, K. Mertins and G. Schmidt (eds.) Handbook on Architectures on Information Systems. Berlin: Springer-Verlag

Nyhuis, P. (1994) Logistic Operating Curves - a Comprehensive Method of Rating Logistic Potentials. In Proceedings of EURO XIII / OR36, University of Strathclyde, Glasgow.

VIMIMS Consortium. Virtual Institute for the Modelling of Industrial Manufacturing Systems (VIMIMS). Proposal submitted to MINERVA action of the European Commission, March 2000.

Wiehndahl, H.-P. (1987) A New Control System for the Production Flow on the Basis of the Throughput Diagram. Manufacturing Systems, 16, 1, 57-65

\section{BIOGRAPHY}

Marco Garetti is professor of industrial technology in the Department of Management, Economics and Industrial Engineering, Politecnico di Milano, Italy. He is a member of WG 5.7 (Integrated Production Management) and WG 5.12 of IFIP and a member of the editorial board of Production Planning and Control by Taylor and Francis. His fields of interest are design of production systems, modelling and simulation of manufacturing systems, production management, logistics and intelligent manufacturing systems. He has been project leader in many research projects at national and international level. He has published four books and some sixty papers. 